Psychother Psychosom

DOI: $10.1159 / 000487599$

\section{Pretreatment Cardiac Vagal Tone Predicts Dropout from and Residual Symptoms after Exposure Therapy in Patients with Panic Disorder and Agoraphobia}

Julia Wendt ${ }^{\mathrm{a}}$ Alfons O. Hamm ${ }^{\mathrm{a}}$ Christiane A.Pané-Farréa Julian F. Thayer ${ }^{\mathrm{b}}$ Alexander Gerlach ${ }^{\mathrm{c}}$ Andrew T. Gloster ${ }^{\mathrm{d}}$ Thomas Lang e,f Sylvia Helbig-Lang ${ }^{f}$ Paul Paulig Thomas Fydrich $^{\mathrm{h}}$ Andreas Ströhle $\mathrm{i}^{\mathrm{i}}$ Tilo Kircher ${ }^{\mathrm{j}}$ Volker Arolt ${ }^{\mathrm{k}}$ Jürgen Deckert' Hans-Ulrich Wittchen ${ }^{\mathrm{m}}$ Jan Richter ${ }^{\mathrm{a}}$

${ }^{a}$ Department of Psychology, University of Greifswald, Greifswald, Germany; ${ }^{b}$ Department of Psychology, Ohio State University, Columbus, OH, USA; ' Department of Psychology, University of Cologne, Cologne, Germany; ${ }^{\text {d Department of }}$ Psychology, University of Basel, Basel, Switzerland; ${ }^{\mathrm{e}}$ ChristophDornier Foundation for Clinical Psychology, Bremen, Germany; fDepartment of Psychology, University of Hamburg, Hamburg, Germany; ${ }^{9}$ Department of Psychology, University of Würzburg, Würzburg, Germany; ${ }^{\text {h}}$ Department of Psychology, Humboldt University of Berlin, Berlin, Germany; 'Department of Psychiatry and Psychotherapy, Charité - Universitätsmedizin Berlin, Berlin, Germany; 'Department of Psychiatry and Psychotherapy, Philipps-University Marburg, Marburg, Germany; ${ }^{\mathrm{k}}$ Department of Psychiatry, University of Münster, Münster, Germany; 'Department of Psychiatry, Psychosomatics and Psychotherapy, University of Würzburg, Würzburg, Germany; ${ }^{m}$ Department of Psychology, Technische Universität Dresden, Dresden, Germany

Exposure therapy is a well-established and effective treatment for pathological anxiety. However, high dropout and nonresponder rates call for the investigation of individual differences that mediate treatment response [1]. Among physiological individual differences, cardiac vagal tone (CVT) seems a promising candidate to predict treatment response: CVT has been linked to cognitive, affective, social, and health functioning levels [2] and low CTV has been found in a variety of anxiety disorders [3]. To test the association between CVT and treatment response, we followed two lines of argument. First, low CVT has been associated with a deficit in extinction learning which is considered an underlying mechanism of exposure therapy [e.g., 4]. Given that pathological anxiety is also associated with an extinction learning deficit [5], CVT may qualify as a factor moderating this association. That is, anxiety patients with low CVT may show less extinction during exposure therapy and, thus, may more likely show residual symptoms. Second, CVT has been linked to self-regulation capacity [2] and, thus, may impact the likelihood of dropout from exposure therapy during which a patient's self-regulation capacity is extraordinarily challenged.

Accordingly, we investigated the association between pretreatment CVT and residual symptoms after exposure therapy and dropout from exposure therapy in a sample of 228 patients with panic disorder and agoraphobia (PD/AG). These PD/AG patients underwent a standardized and manualized treatment focusing on exposure in vivo in a randomized controlled trial carried out in multiple centers $[6,7]$. For the current research question, patients were grouped into those who were (1) fully recovered or (2) showed residual symptoms according to clinician ratings after completion of treatment, and (3) treatment dropouts. Clinician ratings were standardized using the Clinical Global Impression Scale (CGI) that measures the overall severity of a disorder with scores that range between 1 (no disorder) and 7 (among the most severely ill patients). The assessment of pretreatment CVT as indexed by heart rate variability (HRV) was based on electrocardiogram data collected during a relaxation period of a highly standardized Behavioral Avoidance Test (BAT [8]) prior to therapy. The root mean square of successive differences (RMSSD) and high-frequency (HF) HRV (frequency band: $0.15-0.40 \mathrm{~Hz}$ ) were determined using HRV analysis (see online suppl. material for more information about participants, treatment, outcome assessment, HRV assessment, and data analysis; for all online suppl. material, see www. karger.com/doi/10.1159/000487599).

Thirty-nine patients (17.1\%) dropped out from therapy prior to postassessment. In patients completing treatment, postassessment CGI scores lower than 3 indicated no more clinically relevant symptoms (i.e., full recovery) in 28 patients $(12.3 \%)$, and CGI scores of 3 or more indicated residual symptoms in 161 patients (70.6\%). Neither the group with residual symptoms nor the treatment dropout group differed from the fully recovered group with regard to baseline levels of symptom severity (see online suppl. Table S1). As shown in Figure 1, however, the groups differed with regard to pretreatment HRV. The group with residual symptoms had lower pretreatment HRV than the fully recovered group: RMSSD: $t(187)=2.27, p=0.024$; HF-HRV: $t(187)=2.35, p=0.020$. Likewise, the treatment dropout group had lower pretreatment HRV than the fully recovered group: RMSSD: $t(65)=2.69, p=$ 0.009 ; HF-HRV: $t(65)=2.33, p=0.023$ ).

To predict residual symptoms, binary logistic regressions were conducted using either RMSSD or HF-HRV scores to indicate HRV together with sex and age as well as CGI scores as indicators of baseline symptom severity. Fully recovered patients served as a reference group. The Wald criterion indicated that only HRV made a significant contribution to predict residual symptoms: RMSSD: $4.27, p=0.039$; HF-HRV: $4.25, p=0.039$ (see online suppl. Table S2 and text). The same setup for binary logistic regressions was applied to predict treatment dropout as opposed to full recovery and, again, the Wald criterion indicated that only HRV made a significant contribution to predict treatment dropout:

\section{KARGER}

(C) 2018 S. Karger AG, Basel

E-Mail karger@karger.com

www.karger.com/pps
Julia Wendt

Department of Biological and Clinical Psychology, University of Greifswald Franz-Mehring-Strasse 47 DE-17487 Greifswald (Germany)

Greifswald, Germany 


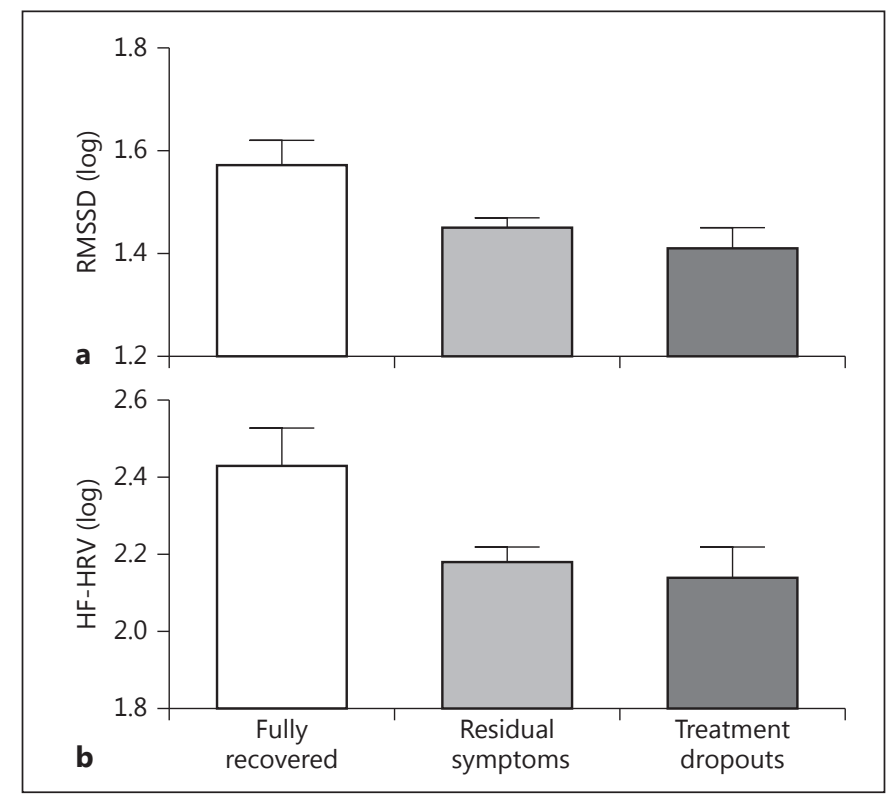

Fig. 1. Pretreatment levels of heart rate variability (HRV) in the fully recovered group, the group with residual symptoms, and the treatment dropout group. Bar charts represent means and standard errors of logarithmized values from the root mean square of successive differences (RMSSD; a) and high-frequency (HF)HRV (b).

RMSSD: 4.72, $p=0.030$; HF-HRV: $3.40, p=0.065$ (see online suppl. Table S3 and text).

These findings indicate that PD/AG patients with low pretreatment CVT are more likely to show residual symptoms after completing exposure-focused therapy. Previous research showed that low CVT is associated with a deficit in safety learning during fear extinction training [e.g., 4]. Both CVT [9] and extinction learning [10] are mediated by inhibitory pathways from the prefrontal cortex to brainstem regions mediated by the amygdala. According to the model of neurovisceral integration [9], this inhibitory corticosubcortical network enables the organism's adaptation to changing environments (e.g., to the absence of an expected threat) by integrating autonomic, attentional, and affective systems. Thus, $\mathrm{PD} / \mathrm{AG}$ patients with lower levels of prefrontal cortex activity as indexed by CVT may experience less effective fear extinction and, thus, may more likely show residual symptoms after completing exposure-focused therapy.

In addition, low pretreatment CVT also predicted treatment dropout in the present study. Entering an exposure situation in which a patient confronts what they most fear (up to and including in some cases the fear of dying) and have long since avoided certainly demands high levels of self-regulation from PD/AG patients. Patients with particularly low pretreatment CVT may have lower levels of self-regulatory capacity and, therefore, more pronounced difficulties in entering and/or remaining in a feared situation. This assumption is further strengthened by the finding that pretreatment CVT is particularly low in patients stating motivational reasons for dropout, e.g., "too afraid to enter exposure situation" (see online suppl. Fig. S1 and text). Thus, PD/AG patients with low
CVT may benefit from increased guidance by the therapist during exposure exercises [8]. Indeed, results from this clinical trial showed that patients who are accompanied by a therapist profit more from exposure therapy than those without guidance [7], especially if pronounced situational avoidance behavior has to be overcome during treatment [8]. In the case of CVT, such tailored treatments are feasible for the practitioner because CVT can be increased by different interventions such as meditation, exercise training, or biofeedback. In conclusion, PD/AG patients with low CVT may benefit from the implementation of such low-intensity interventions prior to undergoing exposure therapy.

\section{Acknowledgments}

This work is part of the German multicenter trial "Mechanisms of Action in CBT" (MAC). The MAC study is funded by the German Federal Ministry of Education and Research (project No. 01GV0615) as part of the German Federal Ministry of Education and Research Psychotherapy Research Funding Initiative. J. Wendt was supported by a grant provided by the German Research Foundation (DFG; WE 5873/1-1).

The ISRCTN registry provided the following study: "Improving cognitive behavioural therapy for panic by identifying the active ingredients and understanding the mechanisms of action: a multicentre study" (http://www.isrctn.com/ISRCTN80046034; 040203-17).

Additional site directors in the randomized clinical trial component of the MAC program are G.W. Alpers (Würzburg) and L. Fehm (Berlin-Adlershof).

Acknowledgements and staff members by site: Greifswald (coordinating site for psychophysiology): Christiane A. Pané-Farré, Jan Richter, Susan Richter, and Matthias von Rad; Berlin - Charité (coordinating center for experimental pharmacology): Harald Bruhn, Anja Siegmund, Meline Stoy, and Andre Wittmann; Berlin-Adlershof: Irene Schulz; Münster (overall MAC program coordination, genetics and functional neuroimaging): Andreas Behnken, Katharina Domschke, Adrianna Ewert, Carsten Konrad, Bettina Pfleiderer, and Peter Zwanzger; Münster (coordinating site for psychophysiology and subtyping): Judith Eidecker, Swantje Koller, Fred Rist, and Anna Vossbeck-Elsebusch; Marburg/Aachen (coordinating center for functional neuroimaging): Barbara Drüke, Sonja Eskens, Thomas Forkmann, Siegfried Gauggel, Susan Gruber, Andreas Jansen, Thilo Kellermann, Isabelle Reinhardt, and Nina Vercamer- Fabri; Dresden (coordinating site for data collection, analysis, and the randomized clinical trial): Franziska Einsle, Christine Fröhlich, Andrew T. Gloster, Christina Hauke, Simone Heinze, Michael Höfler, Ulrike Lueken, Peter Neudeck, Stephanie Preiss, and Dorte Westphal; Würzburg Psychiatry Department (coordinating center for genetics): Andreas Reif; Würzburg Psychology Department: Julia Dürner, Hedwig Eisenbarth, Antje B.M. Gerdes, Harald Krebs, Paul Pauli, Silvia Schad, and Nina Steinhäuser; Bremen: Veronika Bamann, Sylvia Helbig-Lang, Anne Kordt, Pia Ley, Franz Petermann, and EvaMaria Schröder. Additional support was provided by the Coordinating Center for Clinical Studies in Dresden (CCS Dresden): Xina Grählert and Marko Käppler.

\section{Statement of Ethics}

The Randomized Clinical Trial project was approved by the Ethics Committee of the Medical Faculty of the Technical University of Dresden (EK 164082006). 


\section{Disclosure Statement}

J. Deckert received speaker honoraria from Janssen, Bristol Myers-Squibb, Wyeth, Lundbeck, Astra-Zeneca, and Pfizer, and grant support from Medice, Novartis, and Alkermes.

A. Ströhle received research funding from the German Federal Ministry of Education and Research, the European Commission (FP6), and Lundbeck, and speaker honoraria from Pfizer, Eli Lilly \& Co., Wyeth, Lundbeck, AstraZeneca, Boehringer Ingelheim, Bristol-Myers Squibb, and UCB. A. Ströhle was the recipient of educational grants from the Stifterverbandfür die Deutsche Wissenschaft, the Berlin Brandenburgische Akademie der Wissenschaften, the Boehringer Ingelheim Fonds, and the Eli Lilly International Foundation.

T. Kircher received fees for educational programs from Janssen-Cilag, Eli Lilly, Servier, Lundbeck, Bristol Myers-Squibb, Pfizer, and Astra-Zeneca, as well as travel support/sponsorship for congresses from Servier, speaker's honoraria from Janssen-Cilag, and research grants from Pfizer and Lundbeck.

$\mathrm{V}$. Arolt is member of advisory boards and/or gave presentations for the following companies: Astra-Zeneca, Janssen-Organon, Eli Lilly, Lundbeck, Pfizer, Servier, and Wyeth. He also received grants from Astra-Zeneca, Lundbeck, and Wyeth. He chaired the committee for the "Wyeth Research Award Depression and Anxiety." These cooperations have no relevance to the work covered in this paper.

All other authors report no biomedical financial interests or potential conflicts of interest.

\section{Author Contributions}

Principal investigators (PI) with respective areas of responsibility in the MAC study are V. Arolt (Münster: overall MAC program coordination), H.-U. Wittchen (Dresden: PI for the randomized clinical trial and manual development), A.O. Hamm (Greifswald: PI for psychophysiology), A. Gerlach (Münster: PI for psychophysiology and panic subtypes), A. Ströhle (Berlin: PI for experimental pharmacology), T. Kircher (Marburg: PI for functional neuroimaging), and J. Deckert (Würzburg: PI for genetics).

All PI take responsibility for the integrity of the respective study data and their components. All authors and co-authors had full access to all study data. Data analysis and manuscript preparation were completed by the authors and co-authors of this article, who take responsibility for its accuracy and content.

\section{References}

1 Lueken U, Zierhut KC, Hahn T, Straube B, Kircher T, Reif A, Richter J, Hamm A, Wittchen H-U, Domschke K: Neurobiological markers predicting treatment response in anxiety disorders: a systematic review and implications for clinical application. Neurosci Biobehav Rev 2016;66: 143-162.

2 Laborde S, Mosley E, Thayer JF: Heart rate variability and cardiac vagal tone in psychophysiological research - recommendations for experiment planning, data analysis, and data reporting. Front Psychol 2017;8: 213.

-3 Chalmers JA, Quintana DS, Abbott MJ-A, Kemp AH: Anxiety disorders are associated with reduced heart rate variability: a meta-analysis. Front Psychiatry 2014;5:80.

4 Wendt J, Neubert J, Koenig J, Thayer JF, Hamm AO: Resting heart rate variability is associated with the inhibition of conditioned fear. Psychophysiology 2015;52:1161-1166.

5 Duits P, Cath DC, Lissek S, Hox JJ, Hamm AO, Engelhard IM, van den Hout MA, Baas JM: Updated meta-analysis of classical fear conditioning in the anxiety disorders. Depress Anxiety 2015;32:239-253.

-6 Gloster AT, Wittchen H-U, Einsle F, Höfler M, Lang T, Helbig-Lang S, Fydrich T, Fehm L, Hamm AO, Richter J, Alpers GW, Gerlach AL, Ströhle A, Kircher T, Deckert J, Zwanzger P, Arolt V: Mechanism of action in CBT (MAC): methods of a multi-center randomized controlled trial in 369 patients with panic disorder and agoraphobia. Eur Arch Psychiatry Clin Neurosci 2009;259(suppl):S155-S166.

-7 Gloster AT, Wittchen H-U, Einsle F, Lang T, Helbig-Lang S, Fydrich T, Fehm L, Hamm AO, Richter J, Alpers GW, Gerlach AL, Ströhle A, Kircher T, Deckert J, Zwanzger P, Höfler M, Arolt V: Psychological treatment for panic disorder with agoraphobia: a randomized controlled trial to examine the role of therapist-guided exposure in situ in CBT. J Consult Clin Psychol 2011;79:406-420.

8 Hamm AO, Richter J, Pané-Farré C, Westphal D, Wittchen H-U, Vossbeck-Elsebusch AN, Gerlach AL, Gloster AT, Ströhle A, Lang T, Kircher T, Gerdes AB, Alpers GW, Reif A, Deckert J: Panic disorder with agoraphobia from a behavioral neuroscience perspective: Applying the research principles formulated by the Research Domain Criteria (RDoC) initiative. Psychophysiology 2016;53:312-322.

-9 Thayer JF, Lane RD: Claude Bernard and the heart-brain connection: further elaboration of a model of neurovisceral integration. Neurosci Biobehav Rev 2009;33:81-88.

10 Milad MR, Quirk GJ: Fear extinction as a model for translational neuroscience: ten years of progress. Annu Rev Psychol 2012;63:129-151. 